\title{
Research Paper: The Electromyographic Feedback and Feedforward Activity of Selected Lower Extrem- ity Muscles During Toe-in Landing in Female Athletes
}

\author{
Sara Pourheidary $^{1}$ D, Rahman Sheikhhoseini $^{* *}$ (D) Farideh Babakhani $^{1}$ (D)
}

1. Department of Corrective Exercise and Sport Injury, Faculty of Physical Education and Sport Sciences, Allameh Tabataba'i University, Tehran, Iran.

\begin{tabular}{|c|c|}
\hline $\begin{array}{l}\text { Use your device toscan } \\
\text { and read the article online }\end{array}$ & ditation Pourheidary S, Sheikhhoseini R, Babakhani F. The Electromyographic Feedback and Feedforward Activity of Se- \\
\hline ariptra & $\begin{array}{l}\text { lected Lower Extremity Muscles During Toe-in Landing in Female Athletes. Physical Treatments. 2019; 9(4):203-210. http:// } \\
\text { dx.doi.org/10.32598/ptj.9.4.203 }\end{array}$ \\
\hline 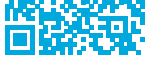 & dol' $h t t p: / / d x . d o i . o r g / 10.32598 / p t j .9 .4 .203$ \\
\hline
\end{tabular}

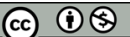

Article info:

Received: 23 Feb 2019

Accepted: 21 Jul 2019

Available Online: 01 Oct 2019

Keywords:

Electromyography,

Volleyball, Lower

extremity, Sports injuries,

Anterior cruciate ligament

\section{A B S T RA C T}

Purpose: Positioning the legs in performing spike technique significantly contributes to the development and prevention of lower limb injuries. The present study aimed to evaluate and compare the feedback and feedforward activaties of selected lower limb muscles during triple jump spike with and without toe-in landing in female volleyball players.

Methods: In this controlled-laboratory study, 15 female elite volleyball players performed triple spike in toe-in and neutral positions. The electrical activity of tibialis anterior, proneus longus, gastrocenemius, vastus medialis, vastus lateralis, semitendinosus, and biceps femoris muscles was recorded using a 16-channel surface electromyography device (Baya Med Company). The Dependent Samples t-test was applied to analyze the obtained data at the $95 \%$ confidence level.

Results: In toe-in landing, the semitendinosus muscle activity $(\mathrm{P}=0.040)$ significantly decreased in the feedforward phase. Besides, in the feedback phase, there were significant increases in tibialis anterior $(\mathrm{P}=0.039)$ and proneus longus $(\mathrm{P}=0.043)$. Eventually, a significant decrease was observed in the medial gastrocenemius $(\mathrm{P}=0.042)$ and biceps femoris muscle $(\mathrm{P}=0.047)$ activities.

Conclusion: Decreased biceps femoris muscle activity during the feedback phase with the toe-in position may be associated with the decreased supportive effect of hamstring muscle, increased anterior knee shear force, and subsequently anterior cruciate ligament injury. Increased activity of leg and ankle muscles may also increase the odds of muscular fatigue and subsequent ankle sprain during toe-in landing.

\section{* Corresponding Author:}

Rahman Sheikhhoseini, PhD.

Address: Department of Corrective Exercise and Sport Injury, Faculty of Physical Education and Sport Sciences, Allameh Tabataba'i University, Tehran, Iran. Phone: +98 (21) 48394134

E-mail: rahman.pt82@gmail.com 


\section{Highlights}

-Landing with a toe-in leg position may increase the risk of ACL injuries.

-Landing with a toe-in leg position may increase the risk of ankle injuries.

\section{Plain Language Summary}

In volleyball, there are three possible foot positions while landing, as follows: toe-in, toe-out, and neutral leg. Previous studies found that the toe-in leg position is associated with the increased internal rotation of the tibia and knee valgus. We aimed to investigate the difference in the feedback and feedforward activities of selected lower limb muscles while landing after triple jump spikes with toe-in or neutral leg positions in female volleyball players. Landing with toe-in or neutral leg positions at different time intervals suggested different effects on volleyball players' lower limb muscles activities. As a result, such change must be considered in muscle activity for selecting the technique used. Our results indicated that toe-in landing in the feedforward phase might increase the odds of anterior shear forces and torsion force in the knees and the ACL injury risk. In the feedback phase, decreased biceps femoris muscle activity in toe-in landing declined hamstring muscle support of the ACL. Besides, the activity of the leg muscles and the positioning of the proneus longus muscle along its length put the ankle in an unstable position. Therefore, players are recommended to perform landing without toe-in positioning and to focus on strengthening hamstring muscles in their training program highly.

\section{Introduction}

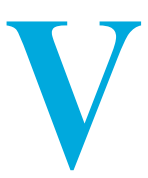

olleyball has become a popular sport since its inception over a hundred years ago [1] and has been practiced by $>800$ million people worldwide [2]. A comprehensive review of epidemiological data suggests that ankle sprains, shoulder overuse, patellar tendonitis, and Anterior Cruciate Lgament (ACL) injuries have been identified as the most prevalent injuries among volleyball players, respectively [3]. Additionally, data from volleyball injuries over many years indicates that $>55 \%$ of injuries occur in the lower extremities during training and competition [4]. Post-injury consequences, like the financial cost of surgery and rehabilitation and psychological matters, necessitated employing injury prevention programs. The extent of lower extremity injuries during jump-landing has been repeatedly reported. Moreover, these injuries reduce the players' efficiency, even after recovery. Therefore, the present research attempted to explore the best performance with the least odds of injury [5].

Volleyball is among the sports with increased ACL injuries in recent decades, due to frequent activities, such as jump-landing and sudden turnaround. In addition, an ACL injury is one of the major injuries requiring longterm rehabilitation. Furthermore, ACL injuries are more prevalent in female athletes, than males; these injuries occur 4-6 times more in elite female athletes compared to their male counterparts [6]. The mechanism of inju- ries in volleyball is divided into contact injuries resulted from collision with an opponent under the net or hitting the ball (more frequent in fingers and ankles) and noncontact injuries; this is due to overuse or repetition of inappropriate techniques (most commonly obreved in the knee, back, \& shoulder) [7].

Landing after jumping in volleyball spikes has been recognized among the most important non-contact mechanisms of ACL injuries [6]. Researchers have further investigated the mechanism of knee ligament injuries. They concluded that the ACL injury occurs with minimal contact mechanism; therefore, it generally has a non-contact mechanism [8]. Numerous studies investigated the impact of jumping and landing in developing ACL injuries among volleyball players $[9,10]$.

Lower extremity injuries are a common problem in volleyball players, especially in females. Moreover, the most common injuries are non-contact in nature (e.g., landing mechanics after a volleyball spike) [11]. In addition, some studies suggested that the leg position may affect lower extremity mechanics while landing. In volleyball, there are three possible foot postures while landing; toe-in, toe-out, and neutral leg positions. Based on a study conducted by Olsen et al. on handball players, the toe-in leg position is associated with the increased internal rotation of the tibia and knee valgus [12].

Furthermore, Padua et al. reported that landing with toe-in and toe-out leg positions of $>30^{\circ}$ may be a signifi- 
cant risk factor for the ACL injuries [13]. Tran et al. also reported that toe-in landing increased hip abduction and internal rotation; knee abduction angles may increase the ACL injury risk [14]. Also, other researches concluded that increased valgus with tibial internal rotation may be considerd as an ACL risk factor [15]. However, toe-in landing is recommended by coaches to prevent the player's foot touch with the volleyball court lines (it may be an error and cause losing points in the matches). Given the pre-discussed cases, such landing could create a high-risk situation for the volleyball players. Moreover, to our-knowledge, no study has been conducted on the electromyographic activity of the lower limb muscles in this type of landing after triple jump spikes; therefore, this study aimed to investigate the difference in the feedback and feedforward activity of selected lower limb muscles while landing after triple jump spikes with toein or neutral leg positions in female volleyball players.

\section{Mterials and Methods}

In this laboratory-controlled study, 15 female volleyball players with a history of playing in the premier league or first division teams of Tehran Province, Iran, voluntarily participated. The study sample was selected using the convenience sampling technique. The study inclusion criteria included an age range of 16-20 years, volleyball training at least 3 days a week for a continuous period of $>3$ years, and a history of national team camp invidtaion or being a member of the Tehran Premier League. The study exclusion criteria were histories of orthopedic surgery in the lower extremities, ACL ligament and the knee meniscus injuries, any condition leading to poor balance, Anterior Talofibular Ligament (ATFL) rupture, and chronic low back pain or acute back pain in the past month, as well as the existence of obvious abnormalities based on the New York criteria (obtaining 1 score every item).

After selecting the study participants, in each test session, the research process was explained; then, informed consent and completed demographic data forms were collected. All study subjects were also assured about their data confidentiality and their right to withdraw from the study at any time. All tests were performed in the exercise laboratory of Allameh Tabataba'i University. The study protocol was approved by the Ethics Committee on Biological Research at the University of Social Welfare and Rehabilitation Sciences (Code: IR.USWR. REC.1397.107). Fist, each study subject performed a 5-min warm-up. Then, the sargent jump test was conducted to obtain $50 \%$ of the vertical jump and adjust the height of the spike jump and landing on the force plate [16]. Before initiating the test, triple jump spike and landing methods were educated to the study subjects; they were requested to practice them three times to get familiarized with and learn the movement. Next, Electromyography (EMG) electrodes were attached to the tibialis anterior, proneus longus, medial gastrocenemius, vastus medialis, vastus lateralis, semitendinosus, and biceps femoris muscles according to the SENIAM (surface EMG for a non-invasive assessment of muscles) protocol. This process was applied as follows: tibialis anterior: in the upper one-third of a line connecting the head of the fibula to the medial malleolus; proneus longus: within the upper quarter of the head of the fibula to the lateral malleolus; lateral gastrocenemius: in the upper one-third of the head of fibula to the center of the posterior aspect of calcaneus; vastus medialis: one-fifth of a line $t$ connecting the anterior superior iliac spine to the anterior aspect of medial collateral ligament; vastus lateralis: one-third of a line connecting the anterior superior iliac spine to the supero-lateral angle of the patella; semitendinosus: half of a line connecting the ischial tuberosity to the medial tibial condyle; and biceps femoris muscle: half of a line connecting the ischial tuberosity to the lateral tibial condyle [17].

Before initiating the test process, the landing foot was determined. For this purpose, the study participants were requested to perform triple jump spike and land on one foot. The foot which the participant felt comfortable to perform the final landing and maintained balance was identified as the landing foot. The EMG activity of Maximum Voluntary Isometric Contraction (MVIC) of each muscle was recorded. Each study subject performed MVIC three times for 10 seconds. The MVIC data were analyzed using LabVIEW.

Furthermore, Root Mean Square (RMS) values were calculated in 3-8 seconds of the three tests, and their mean score was used for data normalization. The MVIC evaluation of muscles was performed as follows. For the biceps femoris, each subject lied in a prone position, and against the maximal resistance, performed the flexion and external isometric rotation of the knee at a $45^{\circ}$ of knee flexion angle; for the semitendinosus, each subject lied in a prone position and against the resistance performed knee flexion and internal isometric rotation [18]. The tibialis anterior was examined in a standing position with the ankle at $90^{\circ}$ while each subject was requested to resist against maximal dorsiflexion motion. For the medial and lateral vastuses, the study subjects were requested to extend the knee against maximal applied resistance while being in a sited position on the chair and the knee flex from $70^{\circ}-90^{\circ}$. For the gastrocenemius, the 
study subjects were requested to sit in long sitting position with full extended knees and the foot sole placed against the wall in $90^{\circ}$ of ankle position; then, they were requested to to perform plantar flexion [19]. Finally, for the proneus longus, the study subjects were positioned in half-sitting, and the dominant ankle was in the normal position (between dorsiflexion and plantar flexion). Resistance was made on the foot against eversion and dorsiflexion, while the examiner made sure that the toes were relaxed [20].

To perform a triple jump spike test, the distance of 70 $\mathrm{cm}$ ahead the force plate was marked with a line on the ground as the last step of the player to place her jump foot on that line. To specify landing direction in neutral or toe-in positions, another mark was drawn on the force plate center with adhesive paper tape; one direction in line with the triple jump spike line and another at the $30^{\circ}$ of its inward line. These two marks helped the study subject to identify their exact landing location on the force plate. The Sargent Jump Test was performed to measure the maximum vertical jump where the subject stood under the scaled jump plate and requested to stand in front of the device and raise her hand as far as possible without raising heel. The middle finger tip height was selected as the zero point of the device and the device was set at this point. The study subject was then requested to make the maximum vertical jump, touch the device with the fingertip at the peak, and land on both legs. This height was recorded as the maximum vertical jump. Each subject jumped three times. Next, after recording the scores, the highest score was selected as the subject's maximum vertical jump [16].

Then, an object was hanged from the ceiling with a height adjusted to $50 \%$ of the maximum vertical jump height. Furthermore, it was placed at the middle distance between the force plate center and the $70 \mathrm{~cm}$ line before the force plate. The triple jump spike starting point was also determined by a line drawn on the ground distanced 3 meters from the force plate. To perform the triple jump spike motion, the study subject first made three triple jump spikes from the identified starting point. Each study subject performed three acceptable triple jump spikes, and the mean values of study variables at these three jumps were recorded. An acceptable triple jump spike comprised a movement onset from the starting point; the first step with the left foot, if she was right-handed (and vice versa), toucheed the marker with the dominant hand, and landed on the line marked at the force plate center. If the subject hopped on the force plate after landing and lost her balance or landed on the wrong foot, the triple jump spike was considered as unaccept- able. For greater accuracy in performing the test with internal ankle rotation, neutral angles and $30^{\circ}$ of internal rotation were determined with the adhesive paper tape on the force plate center. Next, the study subject was requested to attampt to put the foot along the so-called line in each position.

Muscle electrical activity was recorded by an EMG device (16-channel, Baya Med, Iran). The device's pre-amplifiers had a frequency of $4000108 \mathrm{~dB}$ (common mode rejection ration $\mathrm{CMRR}$ ). EMG data were smoothed using low pass $(490 \mathrm{~Hz})$ and high pass $(10 \mathrm{~Hz})$ filters. The RMS was measured by EMG signals to estimate the muscle activity; for the normalization of raw data, the RMS of the selected muscles were divided into their RMS of MVIC [17]. The recorded data of electrical muscle activity were analyzed using the LabVIEW. The moment of initial foot contact was determined using a force plate synchronized with the EMG device; the contact moment was considered as the first moment when the vertical force of the force plate showed a data exceeding $10 \mathrm{~N}$. Each muscle activity at $100 \mathrm{MS}$ before the initial foot contact (feedforward phase) and 100 to $200 \mathrm{MS}$ after the initial foot contact (feedback phase) was extracted and normalized by mean RMS of MVIC. Then, using SPSS, descriptive statistics were used to determine the indices of central tendency and data dispersion. Shapiro-Wilk Test was employed to assess the normal distribution of data. Furthermore, the Dependent Samples $t$-test was was applied to compare muscle activity ratio in two triple jump spike techniques at a $95 \%$ confidence level $(\mathrm{P} \leq 0.05)$.

\section{Results}

The study participants' demographic characteristics, such as weight, height, Body Mass Index (BMI), and age are summarized in Table 1 . For the statistical analysis of data, Shapiro-Wilk Test was used to examine the normality of the data distribution, all of which had a normally-distributed pattern. Therefore, the Paired Samples Dependent t-test was used to compare the study participants. The relevant mean and standard deviation scores of the variables are presented in Table 2.

The Dependent Sanples t-test results for the feedforward phase revealed a statistically significant difference in the activity of the semitendinosus muscle. In other words, this muscle's activity significantly increased in toe-in landing. In the feedback phase, the achieved results indicated a significant difference in the mean activity value of the tibialis anterior, proneus longus, gastrocenemius, and biceps femoris muscles. Furthermore, 
Table 1. Demographic characteristics of the study participants

\begin{tabular}{ccccc}
\hline Variables & No. & Mean \pm SD & Max. & Min. \\
\hline Weight $(\mathrm{kg})$ & 15 & $60.67 \pm 5.85$ & 68.00 & 52.00 \\
Height $(\mathrm{m})$ & 15 & $1.68 \pm 0.42$ & 1.75 & 24.98 \\
$\mathrm{BMI}, \mathrm{kg} / \mathrm{m}^{2}$ & 15 & $21.45 \pm 1.86$ & 22.00 & 19.27 \\
Age $(\mathrm{y})$ & 15 & $18.60 \pm 1.45$ & & 18.00 \\
\hline
\end{tabular}

Table 2. Mean \pm SD scores of the study variables and Dependent Samples t-test results for comparing mean data

\begin{tabular}{|c|c|c|c|c|c|c|}
\hline \multirow{3}{*}{ Muscles } & \multicolumn{3}{|c|}{ Feedback Phase } & \multicolumn{3}{|c|}{ Feedforward Phase } \\
\hline & \multicolumn{2}{|c|}{ Mean \pm SD } & \multirow{2}{*}{$\mathbf{P}$} & \multicolumn{2}{|c|}{ Mean \pm SD } & \multirow{2}{*}{$\mathbf{P}$} \\
\hline & Neutral & Toe-in Position & & Neutral & Toe-in Position & \\
\hline Tibialis anterior & $170.93 \pm 155.34$ & $290.63 \pm 119.36$ & $* 0.039$ & $47.23 \pm 42.18$ & $34.14 \pm 25.24$ & 0.276 \\
\hline Proneus longus & $185.61 \pm 134.82$ & $264.06 \pm 104.46$ & $* 0.043$ & $90.17 \pm 74.21$ & $110.12 \pm 94.22$ & 0.506 \\
\hline Gastrocenemius & $199.89 \pm 141.04$ & $125.66 \pm 96.67$ & $* 0.042$ & $88.84 \pm 73.09$ & $65.49 \pm 47.86$ & 0.294 \\
\hline Vastus medialis & $93.02 \pm 71.77$ & $76.51 \pm 53.58$ & 0.481 & $32.87 \pm 27.61$ & $20.47 \pm 13.94$ & 0.144 \\
\hline Vastus lateralis & $164.69 \pm 124.88$ & $148.51 \pm 112.71$ & 0.716 & $62.80 \pm 47.49$ & $63.23 \pm 45.12$ & 0.979 \\
\hline Biceps femoris & $236.55 \pm 133.72$ & $131.71 \pm 114.58$ & $* 0.047$ & $58.10 \pm 43.15$ & $81.49 \pm 69.85$ & 0.332 \\
\hline Semitendinosus & $200.43 \pm 150.51$ & $120.03 \pm 107.54$ & 0.195 & $49.30 \pm 32.87$ & $22.58 \pm 16.74$ & $* 0.040$ \\
\hline
\end{tabular}

*Observed significant difference.

PHYSICAL TREA $\$ MENTS

the activity of the tibialis anterior muscle and proneus longus significantly increased in the toe-in landing position; however, the activity of gastrocenemius and biceps femoris muscles significantly decreased (Table 2).

\section{Discussion}

The study results suggested a significant difference in the mean value of muscle activity in landing with toe-in and neutral positions in the feedback and feedforward phases. The semitendinosus muscle activity at the feedforward phase was significantly lower than that of landing with toe-in position. A mechanism of noncontact ACL injury is increased quadriceps activity and decreased hamstring muscle activity. If this occurs with internal rotation of the knee, the ACL injury risk may increase. During the feedforward landing phase with the toe-in landing, there was a decrease in hamstring muscles activity along with inward knee rotation, leading to increased ACL injury risk [21]. Previous studies have reported a relationship between hamstring muscle and anterior shear forces.
Additionally, hamstring muscle activation decreased anterior tibial displacement and may reduce demands on ACL [22]. Decreased semitendinosus muscle activity in the landing with the ankle rotated inward can reduce the protective effect [23]. Decreased hamstring muscle activity during toe-in landing may significantly increase ACL injury risk [24]. As a result, the toe-in landing may be a risk factor for the ACL ligament, i.e., consistent with the results of Pourheidary et al. [15] and Tran et al. [14].

In the feedback phase, the activity of biceps femoris and gastrocnemius muscles was less in toe-in landing. Other studies have reported that increased hamstring muscles activity during landing may have a protective effect on the ACL injuries. Therefore, decreased biceps femoris muscle activity during toe-in landing may decrease the supportive effect of this muscle on the ACL; in turn, it indicates an increased risk of injury to the ACL ligament with this landing technique [15]. Both of these muscles have a synergistic role in the ACL; decreased active tibio-femoral stability increased demand on inactive structures, resuting in ACL injury [25]. 
The activity of the anterior tibialis muscles and proneus longus significantly increased in toe-in landing. Increased tibialis anterior and proneus longus muscles activities in toe-in landing at the feedback phase may be due to the position of the ankle that places them in elongated length. Increased activity of these muscles may cause muscle fatigue. Muscle fatigue may alter the movement pattern, increase muscle coactivation, elevate muscle activation, and alter the joint position sense which will increase the odds of lower extremity injury [26]. Muscle fatigue increases the odds of joint injury. When an athlete performs, in a long time, the triple jump spike with toe-in position, the odds of fatigue in the tibialis anterior and proneus longus muscles increases. This may put the ankle joint at risk of further injury [27]. Changes in the activity of the tibialis anterior muscles, proneus longus, and gastrocenemius muscle are essential for ankle instability. An ankle sprain risk is also higher when ankle inversion is increased. Previous studies have revealed that greater activity of proneus longus muscle, compared to the tibialis anterior and gastrocenemius muscles can increase ankle instability injury risk. In this study, the proneus longus muscle was more active than the tibialis anterior and gastrocenemius muscles in toe-in landing. Besides, in the neutral landing, the activity of the gastrocenemius muscles and tibialis anterior muscle was greater than that of the proneus longus muscle, respectively. This may indicate that toe-in landing may increase ankle instability and ankle sprain risks [28].

A limitation of this study was the lack of control over all subjects' nutrition and unequal physical fitness. Similar studies are recommended to be conducted on males and with a larger sample size. The study laboratory conditions differed from the actual conditions; therefore, different results may be obtained if similar research is performed during the training sessions or matches.

\section{Conclusion}

Landing with toe-in or neutral leg positions at different time intervals had different effects on lower limb muscle activity in volleyball players. As a result, it is best to consider this muscle activity change for selecting the technique used. Our results suggested that toe-in landing in the feedforward phase might increase the odds of anterior shear forces and torsion force in the knees and the ACL injury risk. In the feedback phase, a decrease in biceps femoris muscle activity in toe-in landing decreased hamstring muscles' support of the ACL. Additionally, the activity of the leg muscles and the positioning of the proneus longus muscle along its length put the ankle in an unstable position. Therefore, players are recommend- ed to perform their landing without toe-in positioning and to focus on strengthening hamstring muscles in their training program highly.

\section{Ethical Considerations}

\section{Compliance with ethical guidelines}

The study protocol was approved by the Ethics Committee on Biological Research at the University of Social Welfare and Rehabilitation Sciences (Code: IR.USWR. REC.1397.107).

\section{Funding}

This paper was extracted from a dissertation at Allameh Tabataba'i University on Department of sport injuries and corrective exercises in the Winter of 2018.

\section{Authors' contributions}

Original idea, writing the manuscript, protocol development, abstract, and data analysis: Rahman Sheikhhoseini and Sara Pourheidari; Development of the protocol, abstracted data, and prepared the manuscript: all authors.

\section{Conflict of interest}

The Authors declared no acknowledgment.

\section{References}

[1] Tillman MD, Criss RM, Brunt D, Hass CJ. Landing constraints influence ground reaction forces and lower extremity EMG in female volleyball players. Journal of Applied Biomechanics. 2004; 20(1):38-50. [DOI:10.1123/jab.20.1.38]

[2] Cronin JB, Bressel E, Finn LJJoSR. Augmented feedback reduces ground reaction forces in the landing phase of the volleyball spike jump. Journal of Sport Rehabilitation. 2008; 17(2):148-59. [DOI:10.1123/jsr.17.2.148] [PMID]

[3] James LP, Kelly VG, Beckman EM. Injury risk management plan for volleyball athletes. Sports Medicine. 2014 44(9):1185-95. [DOI:10.1007/s40279-014-0203-9] [PMID]

[4] Agel J, Palmieri-Smith RM, Dick R, Woitys EM, Marshall SW Descriptive epidemiology of collegiate women's volleyball injuries: National Collegiate Athletic Association Injury Surveillance System, 1988-1989 through 2003-2004. Journal of Athletic Training. 2007; 42(2):295-302. [PMCID] [PMID]

[5] Miller LS. Examination of hamstring to quadricep muscle ratios in NCAA division I women volleyball athletes [MSc Thesis]. Carbondale, Illinois: Southern Illinois University Carbondale; 2015. 
[6] Zahradnik D, Jandacka D, Holcapek M, Farana R, Uchytil J, Hamill J. Blocking landing techniques in volleyball and the possible association with anterior cruciate ligament injury. Journal of Sports Sciences. 2018; 36(8):955-61. [DOI:10.1080/ 02640414.2017.1346817] [PMID]

[7] Bere T, Kruczynski J, Veintimilla N, Hamu Y, Bahr R. Injury risk is low among world-class volleyball players: 4-year data from the FIVB Injury Surveillance System. British Journal of Sports Medicine. 2015; 2015:094959. [DOI:10.1136/bjsports-2015-094959] [PMID] [PMCID]

[8] Boden BP, Sheehan FT, Torg JS, Hewett TE. Non-contact ACL injuries: Mechanisms and risk factors. Journal of the American Academy of Orthopaedic Surgeons. 2010; 18(9):520-7. [DOI:10.5435/00124635-201009000-00003] [PMID] [PMCID]

[9] Sheikhhoseini R, Alizadeh M-H, Salavati M, O'Sullivan K, Shirzad E, Movahed M. Altered lower limb kinematics during jumping among athletes with persistent low back pain. Annals of Applied Sport Science. 2018; 6(2):23-30. [DOI:10.29252/aassjournal.6.2.23]

[10] Movahed M, Salavati M, Sheikhhoseini R, Arab AM, O'Sullivan K. Single leg landing kinematics in volleyball athletes: A comparison between athletes with and without active extension low back pain. Journal of Bodywork and Movement Therapies. 2019; 23(4):924-9. [DOI:10.1016/j. jbmt.2019.01.012] [PMID]

[11] Olsen OE, Myklebust G, Engebretsen L, Bahr R. Injury mechanisms for anterior cruciate ligament injuries in team handball: A systematic video analysis. American Journal of Sports Medicine. 2004; 32(4):1002-12. [DOI:10.1177/0363546503261724] [PMID]

[12] Padua DA, Marshall SW, Boling MC, Thigpen CA, Garrett Jr WE, Beutler AI. The Landing Error Scoring System (LESS) is a valid and reliable clinical assessment tool of jump-landing biomechanics: the JUMP-ACL study. The American Journal of Sports Medicine. 2009; 37(10):19962002. [DOI:10.1177/0363546509343200] [PMID]

[13] Tran AA, Gatewood C, Harris AH, Thompson JA, Dragoo JL. The effect of foot landing position on biomechanical risk factors associated with anterior cruciate ligament injury. Journal of Experimental Orthopaedics. 2016; 3(1):13. [DOI:10.1186/s40634-016-0049-1] [PMID] [PMCID]

[14] Tavakoli GR. [Comparison of kinematic factors knee joint between jumping whit inward rotation and non rotation tibia in stage landing in man volleyball players (Persian)] [MSc Thesis]. Kerman: Shahid Bahonar University of Kerman; 2016.

[15] Gribble PA, Mitterholzer J, Myers AN. Normalizing considerations for time to stabilization assessment. Journal of Science and Medicine in Sport. 2012; 15(2):159-63. [DOI:10.1016/j.jsams.2011.07.012] [PMID]

[16] Hermens HJ, Freriks B, Disselhorst-Klug C, Rau G. Development of recommendations for SEMG sensors and sensor placement procedures. Journal of Electromyography and Kinesiolog. 2000; 10(5):361-74. [DOI:10.1016/S10506411(00)00027-4]

[17] Pourmahmoudian P, Minoonejad H, Jamshidi AA. [Investigation of the pattern and activity of quadriceps and hamstring muscles in drop from different heights in collegiate male athletes (Persian)]. 2017; 5(4):1-9.
[18] Mahaki M, Mimar R, Shojaedin S, Mahaki B. [The comparsion of the activity pattern of lower limb muscles during single leg drop landing (Persian)]. Health System Research Journal. 2013; 9(9):993-1004.

[19] Konrad P. The ABC of EMG. A practical introduction to kinesiological electromyography. New York: Noraxon USA; 2005.

[20] Hislop H, Avers D, Brown M. Daniels and Worthingham's muscle Testing-E-Book: Techniques of manual examination and performance testing. Amsterdam: Elsevier Health Sciences; 2013.

[21] Shimokochi Y, Shultz SJ. Mechanisms of noncontact anterior cruciate ligament injury. Journal of Athletic Training. 2008; 43(4):396-408. [DOI:10.4085/1062-6050-43.4.396] [PMID] [PMCID]

[22] MacWilliams B, Wilson D, Desjardins J, Romero J, Chao E. Hamstrings cocontraction reduces internal rotation, anterior translation, and anterior cruciate ligament load in weightbearing flexion. Journal of Bone and Joint Surgery. 1999; 17(6):817-22. [DOI:10.1002/jor.1100170605] [PMID]

[23] Withrow TJ, Huston LJ, Wojtys EM, Ashton-Miller JA. Effect of varying hamstring tension on anterior cruciate ligament strain during in vitro impulsive knee flexion and compression loading. The Journal of Bone and Joint Surgery: American volume. 2008; 90(4):815. [DOI:10.2106/ JBJS.F.01352] [PMID] [PMCID]

[24] Fatahi M, Fatahi F. [The relationship between activity level of some lower extremity muscle and anterior shear force during single-leg jump landing (Persian)]. Journal of Research in Rehabilitation Sciences. 2013; 9(4):715-25.

[25] Shultz SJ, Perrin DH, Adams JM, Arnold BL, Gansneder BM, Granata KP. Assessment of neuromuscular response characteristics at the knee following a functional perturbation. Journal of Electromyography and Kinesiology. 2000; 10(3):159-70. [DOI:10.1016/S1050-6411(00)00002-X]

[26] Hatfield G. The effects of quadriceps impairment on lower limb kinematics, kinetics and muscle activation during gait in young adults [MSc. Thesis]. Halifax, Canada: Dalhousie University; 2009.

[27] Paillard T. Effects of general and local fatigue on postural control: A review. Neuroscience \& Biobehavioral Reviews. 2012; 36(1):162-76. [DOI:10.1016/j.neubiorev.2011.05.009] [PMID]

[28] Suda EY, Amorim CF, Sacco Ide C. Influence of ankle functional instability on the ankle electromyography during landing after volleyball blocking. Journal of Electromyography and Kinesiology. 2009; 19(2):e84-93. [DOI:10.1016/j.jelekin.2007.10.007] [PMID] 
This Page Intentionally Left Blank 